\title{
Valuation of Loan Credit Default Swaps Correlated Prepayment and Default Risks with Stochastic Recovery Rate
}

\author{
Yuan $\mathrm{Wu}$ \\ Department of Mathematics, Tongji University \\ 1239 Siping Road, Shanghai 200092, China \\ Tel: +86-21-6515-3240Ｅ-mail: 1.b_forever@yahoo.com.cn \\ Jin Liang (Corresponding author) \\ Department of Mathematics, Tongji University \\ 1239 Siping Road, Shanghai 200092, China \\ Tel: +86-21-6503-9616_E-mail: liang_jin2005@yahoo.com.cn
}

Received: December 6, $2011 \quad$ Accepted: January 5, $2012 \quad$ Published: April 15, 2012

doi: 10.5430/ijfr.v3n2p60

URL: http://dx.doi.org/10.5430/ijfr.v3n2p60

\begin{abstract}
In this paper, we establish an intensity based multi-factor model to value LCDS. The pricing model incorporates the modeling of default, prepayment and recovery risks. Using one factor model, negative correlation between the default and prepayment intensities and positive correlation between the default intensity and the loss given default are described. The interest rate and the house price are chosen as the relevant factors. Under these assumption, a Cauthy problem of PDE is derived, which has a closed-form solution. Based on the solution, numerical examples are provided.
\end{abstract}

Keywords: LCDS, Reduced Form Model, Prepayment Risk, Recovery Risk

\section{Introduction}

Recent years, credit markets have a vigorous development, where new products, such as CDS, CDO, CPDO are brought up. However, with the stronger abilities, these derivatives have also more risky potentials as well. Recent financial crisis makes valuing these credit products more significant.

Loan Credit Default Swaps (LCDS) are almost identical to the standard Credit Default Swaps (CDS) except two features. First, the reference obligation of a LCDS contract is limited on loans; secondly, LCDS contract can be cancelled. This means that correlated prepayment and default should be both considered to price LCDS. Therefore, pricing LCDS is more complicated than doing a standard CDS.

To deal with the risk of LCDS, different types of models have been developed. Zhen Wei (2007) considered a single name LCDS, where using reduced framework, default and prepayment intensities $\lambda_{d}$ and $\lambda_{p}$ were involved. He used a single-factor model with common factor X and correlation coefficient $\rho$ to depict the negative relationship between default and prepayment. However, in his model, the prepayment intensity might be negative, which is meaningless for a single name LCDS. Zhou \& Liang (2010, 2011), Liang \& Wang (2011) developed the model to value a basket LCDS. These models usually require the recovery rate to be constant.

It has already been concluded in several researches that the stochastic recovery rate has a negative correlation with the default intensity. Especially for a loan, the recovery rate is uncertain. However, up to the authors' knowledge, very few papers have involved stochastic recovery rate into pricing credit derivatives. Duffie (2005) introduced stochastic recovery rate theoretically without applications. Peter Dobranszky (2008) built a simple continuous-time stochastic recovery rate to describe the relationship between CDS and LCDS, where he described the negative correlation between default intensity and recovery rate simply by choosing a negative coefficient.

In this paper, based on the models presented in Zhen Wei (2007) and Peter Dobranszky (2008), a pricing model for LCDS under the reduced form framework is established. The negative correlation between default and prepayment risks, and positive correlation between the default intensity and the loss given default are considered in the model. Since all 
rates should be non-negative, we model them as CIR related processes. In this way, we overcome the shortage of Zhen Wei's work, where the prepayment intensity might be negative. To deal with negative correlation, we describe that the negative correlation as an inverse proportion, which was first proposed by Ahn and Gao (1999) and developed by Wu, Jiang \& Liang, (2011) and Qian, Jiang and Xu (2010). Then if one satisfies a CIR process, the other follows inverse CIR process. The problem induces a non-standard partial differential equation which is studied by Hurd \& Kuzneton (2008). Common factors in our 2-factor model for prepayment, default and recovery rates are interest rate and inflection index, which all follow the CIR processes. Under these assumptions, pricing LCDS is modelled where an explicit solution is obtained.

The rest of this paper is organized as follows. In the next section, under some basic notations and assumptions, we establish a pricing model for LCDS. In the third section, we use two-factor model to describe the relationship of default, prepayment, and recovery rate. Then we derive PDE problems and obtain a closed-form of solution for the spread. From the solution, the numerical examples are given in the fourth section.

\section{Notations and Assumptions}

Consider a single-name LCDS contract. The contract is ended before expired time only if a default or a prepayment event happens. Consider a probability space $(\Omega, \mathscr{F}, \mathbf{P})$ and set the following notations:

- $\tau_{d}$ and $\tau_{p}$ : default and prepayment times of the reference of the contract;

- $R(t)$ : recovery rate if the reference defaults;

- $r_{t}$ and $H_{t}$ : risk free interest rate and inflation rate processes;

- $\lambda_{t}^{d}$ and $\lambda_{t}^{p}$ : default and prepayment intensity rates;

- $t_{i}$ and $C_{m}$ : coupon payment date and day count fraction for coupon period $m$.

- $S_{t}$ : LCDS spread, $T$ : maturity time of the contract;

As describing in [11], assuming that $\tau_{d}$ and $\tau_{p}$ are conditional independent, then

$$
\begin{aligned}
& P\left(\tau_{d}>u, \tau_{p}>v\right)=E_{t}\left[e^{-\int_{t}^{u} \lambda_{\tau}^{p} d \tau-\int_{t}^{v} \lambda_{\tau}^{d} d \tau}\right] \\
& \frac{d}{d s} P\left(\tau_{d}>u, \tau_{p}>v\right)=-\left(\lambda_{s}^{p}+\lambda_{t}^{d}\right) E_{t}\left[e^{-\int_{t}^{u}\left(\lambda_{\tau}^{p}+\lambda_{\tau}^{d}\right) d \tau}\right] \\
& \frac{\partial}{\partial u} P\left(\tau_{d}>u, \tau_{p}>v\right)=-E_{t}\left[\lambda_{u}^{d} e^{-\int_{t}^{v} \lambda_{\tau}^{p} d \tau-\int_{t}^{u} \lambda_{\tau}^{d} d \tau}\right]
\end{aligned}
$$

Denote $\tau=\tau^{d} \wedge \tau^{p}$ the first stopping time, that means, the contract survives until $\tau$. On the coupon payment date $t_{m}$, the buyer should pay $S C_{m}$. Supposing the last coupon payment date before $\tau$ is $t_{i}$, the present value of the coupon payment paid till $t_{i}$ is given by

$$
P V_{\text {spread }}(t)=S_{t} C_{m} E_{t}\left[\sum_{t_{i} \geq t} e^{-\int_{t}^{t_{i}} r_{u} d u} 1_{\left\{\tau>t_{i}\right\}}\right]=S_{t} C_{m} E_{t}\left[\sum_{t_{i} \geq t} e^{-\int_{t}^{t_{i}}\left(\lambda_{u}^{p}+\lambda_{u}^{d}+r_{u}\right) d u}\right]
$$

If the first stopping time is during $\left[\mathrm{t}_{\mathrm{i}}, \mathrm{t}_{\mathrm{i}+1}\right]$, the contract is unwound. In this case, by (2), the present value of the coupon accrual. Supposing the last coupon payment date before $\tau$ is unwound. In this case, the present value of the coupon payment paid till $t_{i}$ is given by

$$
\begin{aligned}
& P V_{\text {accrual }}(t)=S_{t} C_{m} E_{t}\left[\sum_{t_{i} \geq t} e^{-\int_{t}^{t_{i}} r_{u} d u} \frac{\tau-t_{i}}{t_{i+1}-t_{i}} 1_{\left\{t v t_{i}<\tau \leq t_{i+1}\right\}}\right] \\
& =-S_{t} \sum_{t_{i} \geq t} \frac{C_{m}}{t_{i+1}-t_{i}} \int_{t v t_{i}}^{t_{i+1}} e^{-\int_{t}^{t_{i}} r_{u} d u}\left(s-t_{i}\right) \frac{d P_{t}(\tau>s)}{d s} d s \\
& =S_{t} C_{m} \sum_{t_{i} \geq t} \frac{1}{t_{i+1}-t_{i}} \int_{t \vee t_{i}}^{t_{i+1}} e^{-\int_{t}^{t_{i}} r_{u} d u}\left(s-t_{i}\right) E_{t}\left[\left(\lambda_{s}^{d}+\lambda_{s}^{d}\right) e^{-\int_{t}^{t_{i}}\left(\lambda_{u}^{p}+\lambda_{u}^{d}+r_{u}\right) d u}\right] d s
\end{aligned}
$$

The buyer stops paying coupons once the prepayment or default occurs. Then the present value of the premium leg is 
given by

$$
P V_{\text {fee }}(t)=S_{t} C_{m} \sum_{t_{i} \geq t}\left(E_{t}\left[e^{-\int_{t}^{t_{i}}\left(\lambda_{u}^{p}+\lambda_{u}^{d}+r_{u}\right) d u}\right]+\frac{1}{t_{i+1}-t_{i}} \int_{t \vee t_{i}}^{t_{i+1}} e^{-\int_{t}^{t_{i}} r_{u} d u}\left(s-t_{i}\right) E_{t}\left[\left(\lambda_{s}^{p}+\lambda_{s}^{d}\right) e^{-\int_{t}^{t_{i}}\left(\lambda_{u}^{p}+\lambda_{u}^{d}\right) d u}\right] d s\right)
$$

On the other hand, using (3), the expected present value of the losses in case of default is:

$$
\begin{aligned}
& P V_{\text {loss }}(t)=E_{t}\left[e^{-\int_{t}^{\tau_{d}} r_{u} d u}\left(1-R\left(\tau_{d}\right)\right) 1_{\left\{\tau_{d}<\min \left(\tau_{p}, T\right)\right\}}\right]=\int_{t}^{T} \int_{t}^{v \wedge T}\left(1-R_{u}\right) e^{-\int_{t}^{u} r_{s} d s} \frac{\partial^{2} P_{t}\left(\tau_{d}>u, \tau_{p}>v\right)}{\partial u \partial v} d u d v \\
& =\left.\int_{t}^{T}\left(1-R_{u}\right) e^{-\int_{t}^{u} r_{s} d s} \frac{\partial P_{t}\left(\tau_{d}>u, \tau_{p}>v\right)}{\partial u}\right|_{v=u} ^{v=T} d u=\int_{t}^{T} E_{t}\left[\left(1-R_{u}\right) e^{-\int_{t}^{u} r_{s} d s} \lambda_{d}^{u} e^{-\int_{t}^{u}\left(\lambda_{p}^{s}+\lambda_{d}^{s}\right) d s} d u\right]
\end{aligned}
$$

Hence, the value of LCDS spread at time $t$ comes:

$$
S_{t}=\frac{\int_{t}^{T} E_{t}\left[\left(1-R_{u}\right) e^{-\int_{t}^{u} r_{s} d s} \lambda_{d}^{u} e^{-\int_{t}^{u}\left(\lambda_{p}^{s}+\lambda_{d}^{s}\right) d s} d u\right]}{C_{m} \sum_{t_{i} \geq t}\left(E_{t}\left[e^{-\int_{t}^{t_{i}}\left(\lambda_{u}^{p}+\lambda_{u}^{d}+r_{u}\right) d u}\right]+\frac{1}{t_{i+1}-t_{i}} \int_{t \vee t_{i}}^{t_{i+1}} e^{-\int_{t}^{t_{i}} r_{u} d u}\left(s-t_{i}\right) E_{t}\left[\left(\lambda_{s}^{p}+\lambda_{s}^{d}\right) e^{-\int_{t}^{t_{i}}\left(\lambda_{u}^{p}+\lambda_{u}^{d}\right) d u}\right] d s\right)}
$$

\section{LCDS price Modeling}

\subsection{Default intensity, prepayment intensity and recovery rates}

As mentioned before, on pricing LCDS, the default and the prepayment risks are negatively correlated. If stochastic recovery rate is involved, it is also negatively correlated with the default risk.

In reality, the factors that affect the default risk, prepayment risk and recovery are complicated. Researchers have shown that among the factors, the interest rate is the most important one. When interest rate decreases, borrowers may refinance their loans, which lead to higher prepayment rate. However, the situation is opposite to the default events, i.e. the probability of default decreases. Another important fact is inflation rate, which affects the default and recovery rates. The higher the inflation rate is, the higher recovery and lower default rate are. To meet these facts, we take two-factor model to describe the relationship of the default risk, prepayment risk and recovery. Interest rate $r_{t}$ and inflation rate $H_{t}$ can serve as the correlated factors of them as follows:

$$
\lambda_{t}^{d}=\rho_{1} r_{1}+\frac{\rho_{4}}{H_{t}}+a_{1} X_{t}^{d}, \quad \lambda_{t}^{p}=\frac{\rho_{2}}{r_{t}}+a_{2} X_{t}^{p}, \quad 1-R_{t}=\rho_{3} e^{-H_{t}}
$$

where $X_{t}^{d}, X_{t}^{p}$ are the specific factor of $\lambda_{t}^{d}, \lambda_{t}^{p}$, and $0<\rho_{1}, \rho_{2}, \rho_{3}, \rho_{4}<1$. We suppose that $r_{t}, X_{t}^{d}, X_{t}^{p}, H_{t}$ are independent. They follow CIR processes as follows:

$$
\begin{aligned}
& d r_{t}=k\left(\theta-r_{t}\right) d t+\sigma \sqrt{r_{t}} d W_{t}^{r}, \\
& d X_{t}^{i}=\kappa_{i}\left(\theta_{i}-X_{t}^{i}\right) d t+\sigma_{i} \sqrt{X_{t}^{i}} d W_{t}^{i}, i=d, p, \\
& d H_{t}=\tilde{k}\left(\tilde{\theta}-H_{t}\right) d t+\tilde{\sigma} \sqrt{H_{t}} d W_{t}^{H},
\end{aligned}
$$

where $\kappa, \theta, \sigma, \kappa_{i}, \theta_{i}, \sigma_{i}, \widetilde{\kappa}, \widetilde{\theta}, \widetilde{\sigma}$ are positive constants; $W_{t}^{r}, W_{t}^{H}, W_{t}^{i}$ are independent standard Brownian motions. Under these assumptions, $r_{t}, X_{t}^{i}, H_{t}$ are mean reverting processes. It is well know that, under the conditions, $2 k \theta>\sigma^{2}, 2 k_{i} \theta_{i}>\sigma_{i}^{2},(i=d, p), 2 \tilde{k} \tilde{\theta}>\tilde{\sigma}^{2}, r_{t}, X_{t}^{i}, H_{t}$ are nonnegative and their boundary at origin are unattainable.

On the other hand, $1 / H_{t}$ follows Inverse CIR (ICIR) process. Let $y_{t}=1 / H_{t}$, by Ito formula:

$$
\frac{d y_{t}}{y_{t}}=\left[\tilde{k}-\left(\tilde{k} \tilde{\theta}-\tilde{\sigma}^{2}\right) y_{t}\right] d t-\tilde{\sigma} \sqrt{y_{t}} d W_{t}^{H}
$$

It has been proved that $y_{t}$ is stable, and it cannot equal 0 or $\infty$ if and only if $2 \tilde{k} \tilde{\theta}>\tilde{\sigma}^{2}, \tilde{\kappa}>0, \tilde{\theta}>0$. Under this 
condition, the origin CIR process $H_{t}$ still remains mean reverting and nonnegative. The situation is the same to $r_{t}$.

\subsection{The solution under CIR process}

Taking equations (5) into equation (4), by noticing that the independence among $r_{t}, X_{t}^{d}, X_{t}^{p}$ and $H_{t}$, the pricing formula (4) turns to:

$$
S_{t}=\frac{\rho_{3} \int_{t}^{T} E_{t}\left[\left(\rho_{1} r_{s}+\frac{\rho_{4}}{H_{s}}+a_{1} X_{t}^{s}\right) e^{-\int_{t}^{s}\left[\left(\rho_{1}+1\right) r_{u}+\frac{\rho_{2}}{r_{u}}+\frac{\rho_{4}}{H_{u}}+a_{1} X_{d}^{u}+a_{2} X_{p}^{u}\right] d u-H_{s}}\right] d s}{V_{M}}
$$

where

$$
\begin{aligned}
& V_{M}=C_{i} \sum_{t_{i} \geq t}\left\{E_{t}\left[e^{-\int_{t}^{t_{i}}\left[\left(\rho_{1}+1\right) r_{u}+\frac{\rho_{2}}{r_{u}}+\frac{\rho_{4}}{H_{u}}+a_{1} X_{d}^{u}+a_{2} X_{p}^{u}\right] d u}\right]\right. \\
& \left.+\frac{1}{t_{i+1}-t_{i}} \int_{t \vee t_{i}}^{t_{i+1}}\left(s-t_{i}\right) E_{t}\left[\left(\rho_{1} r_{s}+\frac{\rho_{2}}{r_{s}}+\frac{\rho_{4}}{H_{s}}+a_{1} X_{d}^{s}+a_{2} X_{p}^{s}\right) e^{-\int_{t}^{s}\left[\left(\rho_{1}+1\right) r_{u}+\frac{\rho_{2}}{r_{u}}+\frac{\rho_{4}}{H_{u}}+a_{1} X_{d}^{u}+a_{2} X_{p}^{u}\right] d u}\right] d s\right\}
\end{aligned}
$$

Observing the equation, we can find the problem can be solved as long as the following three expectations are derived:

$$
\begin{aligned}
& \text { 1. } E_{1}=E_{t}\left[\left(\rho_{1} r_{s}+\frac{\rho_{4}}{H_{s}}+a_{1} X_{t}^{s}\right) e^{-\int_{t}^{s}\left[\left(\rho_{1}+1\right) r_{u}+\frac{\rho_{2}}{r_{u}}+\frac{\rho_{4}}{H_{u}}+a_{1} X_{d}^{u}+a_{2} X_{p}^{u}\right] d u-H_{s}}\right] \\
& =E_{t}\left[\rho_{1} r_{s} e^{-\int_{t}^{s}\left[\left(\rho_{1}+1\right) r_{u}+\frac{\rho_{2}}{r_{u}}\right] d u}\right] E_{t}\left[e^{-\int_{t}^{s} \frac{\rho_{4}}{H_{u}} d u-H_{s}}\right] E_{t}\left[e^{-\int_{t}^{s} a_{1} X_{d}^{u} d u}\right] E_{t}\left[e^{-\int_{t}^{s} a_{2} X_{p}^{u} d u}\right] \\
& +E_{t}\left[\frac{\rho_{4}}{H_{s}} e^{-\int_{t}^{s} \frac{\rho_{4}}{H_{u}} d u-H_{s}}\right] E_{t}\left[e_{s}^{-\int_{t}^{s}\left[\left(\rho_{1}+1\right) r_{u}+\frac{\rho_{2}}{r_{u}}\right] d u}\right] E_{t}\left[e^{-\int_{t}^{s} a_{1} X_{d}^{u} d u}\right] E_{t}\left[e^{-\int_{t}^{s} a_{2} X_{p}^{u} d u}\right] \\
& +E_{t}\left[a_{1} X_{d}^{s} e^{-\int_{t}^{s} a_{1} X_{d}^{u} d u}\right] E_{t}\left[e_{s}^{-\int_{t}^{s}\left[\left(\rho_{1}+1\right) r_{u}+\frac{\rho_{2}}{r_{u}}\right] d u}\right] E_{t}\left[e^{-\int_{t}^{s} a_{2} X_{p}^{u} d u}\right] E_{t}\left[e^{-\int_{t}^{s} \frac{\rho_{4}}{H_{u}} d u-H_{s}}\right] \\
& \text { 2. } E_{2}=E_{t}\left[e^{-\int_{t}^{t_{i}}\left[\left(\rho_{1}+1\right) r_{u}+\frac{\rho_{2}}{r_{u}}+\frac{\rho_{4}}{H_{u}}+a_{1} X_{d}^{u}+a_{2} X_{p}^{u}\right] d u}\right] \\
& =E_{t}\left[e^{-\int_{t}^{t_{i}}\left[\left(\rho_{1}+1\right) r_{u}+\frac{\rho_{2}}{r_{u}}\right] d u}\right] E_{t}\left[e^{-\int_{t}^{t_{i}} a_{1} X_{d}^{u} d u}\right] E_{t}\left[e^{-\int_{t}^{t_{i}} a_{2} X_{p}^{u} d u}\right] E_{t}\left[e^{-\int_{t}^{t_{i}} \frac{\rho_{4}}{H_{u}} d u}\right] \\
& \text { 3. } E_{3}=E_{t}\left[\left(\rho_{1} r_{s}+\frac{\rho_{2}}{r_{s}}+\frac{\rho_{4}}{H_{s}}+a_{1} X_{d}^{s}+a_{2} X_{p}^{s}\right) e^{-\int_{t}^{s}\left[\left(\rho_{1}+1\right) r_{u}+\frac{\rho_{2}}{r_{u}}+\frac{\rho_{4}}{H_{u}}+a_{1} X_{d}^{u}+a_{2} X_{p}^{u}\right] d u}\right] \\
& =E_{t}\left[\left(\rho_{1} r_{s}+\frac{\rho_{2}}{r_{s}}\right) e^{-\int_{t}^{s}\left[\left(\rho_{1}+1\right) r_{u}+\frac{\rho_{2}}{r_{u}}\right] d u}\right] E_{t}\left[e^{-\int_{t}^{s} \frac{\rho_{4}}{H_{u}} d u}\right] E_{t}\left[e^{-\int_{t}^{s} a_{1} X_{d}^{u} d u}\right] E_{t}\left[e^{-\int_{t}^{s} a_{2} X_{p}^{u} d u}\right]
\end{aligned}
$$


$+E_{t}\left[\frac{\rho_{4}}{H_{s}} e^{-\int_{t}^{s} \frac{\rho_{4}}{H_{u}} d u}\right] E_{t}\left[e^{-\int_{t}^{s}\left[\left(\rho_{1}+1\right) r_{u}+\frac{\rho_{2}}{r_{u}}\right] d u}\right] E_{t}\left[e^{-\int_{t}^{s} a_{1} X_{d}^{u} d u}\right] E_{t}\left[e^{-\int_{t}^{s} a_{2} X_{p}^{u} d u}\right]$
$+E_{t}\left[a_{1} X_{d}^{s} e^{-\int_{t}^{s} a_{1} X_{d}^{u} d u}\right] E_{t}\left[e^{-\int_{t}^{s} \frac{\rho_{4}}{H_{u}} d u}\right] E_{t}\left[e^{-\int_{t}^{s}\left[\left(\rho_{1}+1\right) r_{u}+\frac{\rho_{2}}{r_{u}}\right] d u}\right] E_{t}\left[e^{-\int_{t}^{s} a_{2} X_{p}^{u} d u}\right]$
$+E_{t}\left[a_{2} X_{p}^{s} e^{-\int_{t}^{s} a_{2} X_{p}^{u} d u}\right] E_{t}\left[e^{-\int_{t}^{s} \frac{\rho_{4}}{H_{u}} d u}\right] E_{t}\left[e^{-\int_{t}^{s}\left[\left(\rho_{1}+1\right) r_{u}+\frac{\rho_{2}}{r_{u}}\right] d u}\right] E_{t}\left[e^{-\int_{t}^{s} a_{1} X_{d}^{u} d u}\right]$

Thus, we just need to solve the expectations in the following types:

$E_{t}\left[X_{\tau} e^{-a \int_{t}^{\tau} X_{u} d u}\right], E_{t}\left[e^{-a \int_{t}^{\tau} X_{u} d u}\right], E_{t}\left[X_{\tau}^{\omega_{1}} e^{-\omega_{2} X_{\tau}} e^{-\int_{t}^{\tau}\left(d_{1} X_{u}+\frac{d_{2}}{X_{u}}\right) d u}\right]$

where $\left(X_{t}\right)_{t \geq 0}$ follows CIR process. Then $E_{1}, E_{2}, E_{3}$ can be expressed by the above three expectations, then the equation (9) is solved.

Let us solve the three expectations of (11). From the method introduced from of Wei (2007), first, define

$$
I_{t, \tau}^{X}(a, z)=E_{t}\left[e^{-a \int_{t}^{\tau} X_{u} d u+z X_{\tau}}\right] \text {, }
$$

then

$$
E_{t}\left[X_{\tau} e^{-a \int_{t}^{\tau} X_{u} d u}\right]=\left.\partial_{z} I_{t, \tau}^{X}(a, z)\right|_{z=0}, E_{t}\left[e^{-a \int_{t}^{\tau} X_{u} d u}\right]=\left.I_{t, \tau}^{X}(a, z)\right|_{z=0}
$$

Let $u(x, t)=I_{t, \tau}^{X}(a, z)=E_{t}\left[e^{-a l_{t}^{\tau} X_{u} d u+z X_{\tau}}\right]$, by Feynman-Kac formula, $u(x, t)$ is the solution of the following PDE problem:

$\left\{\begin{array}{l}u_{t}+\kappa(\theta-x) u_{x}+\frac{1}{2} \sigma^{2} x u_{x x}-a x u=0, \\ u(x, \tau)=e^{z x}\end{array}\right.$

Suppose that this problem has affine structure solution $u(x, t)=e^{A(t)+x B(t)}$, then $A(t)$ and $B(t)$ can be solved from ODE problems to obtain:

$$
\begin{aligned}
& A(t)=-\frac{2 \kappa \theta}{\sigma^{2}} \cdot \ln \left[\frac{\left(\sigma^{2} z-2 d_{2}\right) e^{-d_{1}(\tau-t)}+\left(2 d_{1}-\sigma^{2} z\right) e^{-d_{2}(\tau-t)}}{\left(\sigma^{2} z-2 d_{2}\right)+\left(2 d_{1}-\sigma^{2} z\right)}\right], \\
& B(t)=\frac{2}{\sigma^{2}} \cdot \frac{\left(\sigma^{2} z-2 d_{2}\right) d_{1} e^{-d_{1}(\tau-t)}+\left(2 d_{1}-\sigma^{2} z\right) d_{2} e^{-d_{2}(\tau-t)}}{\left(\sigma^{2} z-2 d_{2}\right) e^{-d_{1}(\tau-t)}+\left(2 d_{1}-\sigma^{2} z\right) e^{-d_{2}(\tau-t)}} .
\end{aligned}
$$

where

$$
d_{1}=\frac{\kappa-\sqrt{\kappa^{2}+2 a \sigma^{2}}}{2}, d_{2}=\frac{\kappa+\sqrt{\kappa^{2}+2 a \sigma^{2}}}{2}
$$

Thus the solution of (12) is:

$I_{t, \tau}^{X}(a, z)=\left[\frac{\left(\sigma^{2} z-2 d_{2}\right) e^{-d_{1}(\tau-t)}+\left(2 d_{1}-\sigma^{2} z\right) e^{-d_{2}(\tau-t)}}{\left(\sigma^{2} z-2 d_{2}\right)+\left(2 d_{1}-\sigma^{2} z\right)}\right]^{\frac{-2 \kappa \theta}{\sigma^{2}}} e^{\frac{2 x}{\sigma^{2}} \frac{\left(\sigma^{2} z-2 d_{2}\right) d_{1} e^{-d_{1}(\tau-t)}+\left(2 d_{1}-\sigma^{2} z\right) d_{2} e^{-d_{2}(\tau-t)}}{\left(\sigma^{2} z-2 d_{2}\right) e^{-d_{1}(\tau-t)}+\left(2 d_{1}-\sigma^{2} z\right) e^{-d_{2}(\tau-t)}}}$.

From (13), it comes 


$$
\begin{aligned}
& E_{t}\left[X_{\tau} e^{-a \int_{t}^{\tau} X_{u} d u}\right]=\left(\kappa \theta \frac{e^{-d_{1}(\tau-t)}-e^{-d_{2}(\tau-t)}}{d_{2} e^{-d_{1}(\tau-t)}-d_{1} e^{-d_{2}(\tau-t)}}+x \frac{\left(d_{1}-d_{2}\right)^{2} e^{-\kappa(\tau-t)}}{\left(-d_{2} e^{-d_{1}(\tau-t)}+d_{1} e^{-d_{2}(\tau-t)}\right)^{2}}\right) \\
& \cdot\left(\frac{d_{2} e^{-d_{1}(\tau-t)}-d_{1} e^{-d_{2}(\tau-t)}}{d_{2}-d_{1}}\right)^{\frac{-2 \kappa \theta}{\sigma^{2}}} e^{\frac{2 x}{\sigma^{2}} \frac{d_{1} d_{2}\left(e^{-d_{1}(\tau-t)}-e^{-d_{2}(\tau-t)}\right)}{d_{2} e^{-d_{1}(\tau-t)}-d_{1} e^{-d_{2}(\tau-t)}}} .
\end{aligned}
$$

And

$$
E_{t}\left[e^{-a \int_{t}^{\tau} X_{u} d u}\right]=\left(\frac{d_{2} e^{-d_{1}(\tau-t)}-d_{1} e^{-d_{2}(\tau-t)}}{d_{2}-d_{1}}\right)^{\frac{-2 \kappa \theta}{\sigma^{2}}} e^{\frac{2 x}{\sigma^{2}} \frac{d_{1} d_{2}\left(e^{-d_{1}(\tau-t)}-e^{-d_{2}(\tau-t)}\right)}{d_{2} e^{-d_{1}(\tau-t)}-d_{1} e^{-d_{2}(\tau-t)}}}
$$

where $d_{1}$ and $d_{2}$ are defined in (15).

Now, let us solve the third expectation of (11). Hurd and Kuznetsov (2006) have proved that if $X_{t}$ follows CIR process, this expectation can be derived in closed form in terms of a confluent hypergeometric function.

Define $v(x, t)=E_{t}\left[f(x) e^{-\int_{t}^{s}\left(d_{1} x+\frac{d_{2}}{x}\right) d u}\right]$, using Feynman-Kac formula, $v(x, t)$ satisfies the following PDE problem:

$$
\left\{\begin{array}{l}
v_{t}+\kappa(\theta-x) v_{x}+\frac{1}{2} \sigma^{2} x v_{x x}-\left(d_{1} x+\frac{d_{2}}{x}\right) v=0, \\
v(x, s)=f(x)
\end{array}\right.
$$

Let $v(x, t)=e^{-a x} x^{b} w(x, t)$, where $a, b$ are coefficients to be determined, and then $w(x, t)$ satisfies the following equation:

$$
\begin{aligned}
& w_{t}+\left[\kappa \theta+\sigma^{2} b-\left(\sigma^{2} a+\kappa\right) x\right] w_{x}+\frac{1}{2} \sigma^{2} x w_{x x} \\
& +\left[\left(\frac{1}{2} a^{2} \sigma^{2}+a \kappa-d_{1}\right) x+\left(\frac{\sigma^{2} b(b-1)}{2}+b \kappa \theta-d_{2}\right) \frac{1}{x}-\left(\sigma^{2} a b+a \kappa \theta+\kappa b\right)\right] w=0
\end{aligned}
$$

Let $a^{2} \sigma^{2} / 2+a \kappa-d_{1}=0$, and $\sigma^{2} b(b-1) / 2+b \kappa \theta-d_{2}=0$ to solve $a$ and $b$. Now let $w(x, t)=e^{-\left(\sigma^{2} a b+a \kappa \theta+\kappa b\right)} u(x, t)$, then $u(x, t)$ satisfies the following PDE problem:

$$
\left\{\begin{array}{l}
u_{t}+\kappa^{\prime}\left(\theta^{\prime}-x\right) u_{x}+\frac{1}{2} \sigma^{2} x u_{x x}=0, \\
u(x, s)=f(x) e^{a x} x^{-b}
\end{array}\right.
$$

where $\kappa^{\prime}=\sigma^{2} a+\kappa, \theta^{\prime}=\left(\sigma^{2} b+\kappa \theta\right) /\left(\sigma^{2} a+\kappa\right)$. The solution is:

$u(x, t, s)=\int_{0}^{\infty} f(y) e^{a y} y^{-b} \cdot G(y, s, x, t) d y$

where

$$
G(y, s, x, t)=c \cdot e^{-c(\mu+v)} \cdot\left(\frac{\mu}{v}\right)^{\frac{q}{2}} \cdot I_{q}\left(2(\mu v)^{\frac{1}{2}}\right)
$$

And

$$
c=\frac{2 \kappa^{\prime}}{\sigma^{2}\left(1-e^{-\kappa^{\prime}(s-t)}\right)}, q=\frac{2 \kappa^{\prime} \theta^{\prime}}{\sigma^{2}}-1, \mu=c x e^{-\kappa(s-t)}, v=c y, I_{q}\left(2(\mu v)^{\frac{1}{2}}\right)=(\mu v)^{\frac{q}{2}} \sum_{k=0}^{\infty} \frac{(\mu v)^{k}}{k ! \Gamma(q+k+1)} .
$$

$f(y)$ can be expressed by $e^{a^{\prime} y} y^{-b^{\prime}}$, so

$u(x, t, s)=\int_{0}^{\infty} f(y) e^{a y} y^{-b} \cdot G(y, s, x, t) d y=\int_{0}^{\infty} e^{\alpha y} y^{-\beta} \cdot G(y, s, x, t) d y$ 
$=\frac{c^{q+1} e^{-\mu}}{(c-\alpha)^{q-\beta+1}} \frac{\Gamma(q-\beta+1)}{\Gamma(q+1)} M\left(q-\beta+1, q+1, \frac{c \mu}{c-\alpha}\right)$,

where $\alpha$ and $\beta$ depend on $f(y), \quad M(\vartheta, v, z)=\sum_{k=0}^{\infty} \frac{(\vartheta)_{k}}{k !(v)_{k}} z^{k}$ is confluent hypergeometric function and $(\vartheta)_{k}=\vartheta(\vartheta+1) \cdots(\vartheta+k-1), k>0,(\vartheta)_{0}=1$.

\section{Numerical Examples}

So far, we have derived the solution of the pricing equation (4). Now using the solution, we give some numerical examples under the model, where we take $t=0$. And the parameters are shown as follows:

$$
\begin{aligned}
& \theta_{r}=0.03, \theta_{d}=0.03, \theta_{h}=0.03, \theta_{p}=0.03, \sigma_{r}=0.05, \sigma_{d}=0.05, \sigma_{p}=0.05, \sigma_{h}=0.05, \\
& \kappa_{r}=0.15, \kappa_{d}=0.15, \kappa_{p}=0.15, \kappa_{h}=0.15, x_{0}^{d}=0.02, r_{0}=0.02, x_{0}^{p}=0.02, h_{0}=0.02, \\
& \rho_{1}=2, \rho_{2}=0.002, \rho_{3}=0.3, \rho_{4}=0.002, a_{1}=2, a_{2}=1 .
\end{aligned}
$$

Figure 1 shows the relationship of LCDS spread with $r_{0}, \theta_{r}, \kappa_{r}$ and $\sigma_{r}$ respectively. It is obvious that the LCDS spread increases as $r_{0}, \theta_{r}$ or $\sigma_{r}$ increases. That is, the LCDS spread increases as $r$ increases, because $\lambda_{d}$ increases and $\lambda_{p}$ decreases when $r$ increases. It also shows that the effects of $r_{0}$ and $\theta_{r}$ play more important roles than ones of $\sigma_{r}$ and $\kappa_{r}$.

Figure 2 shows the relationship of LCDS spread with $h_{0}, \theta_{h}, \kappa_{h}$ and $\sigma_{h}$ respectively. The figure indicates that the LCDS spread decreases as $h_{0}, \theta_{h}$ or $\sigma_{h}$ increases. That is, the LCDS spread decreases as \$h\$ increases, because $\lambda_{d}$ decreases and $\lambda_{p}$ increases when $h$ increases. And it can be observed from Figure 1 and Figure 2 that the effect of the inflection plays a more important role than that of the interest.

Figure 3 indicates that the trend of LCDS spread with stochastic recovery rate is quite different from the one with the constant recovery rate. It suggests that the stochastic property of recovery rate gives the LCDS Spread volatility and the LCDS spread becomes more varying. We also find that the LCDS spread has a hump type shape which is similar to the one of many stochastic interest products.

\section{Conclusion}

In this paper, pricing LCDS is considered. We develop an intensity based multi-factor model, which incorporates the joint modeling of default, prepayment and recovery risks. We identified two correlations as risk factors that may explain the correlation among default intensity, prepayment intensities and recovery rates. The two common factors are assumed to be interest and inflection rates, which follow CIR process. This structure endure that the rates are non-negative and satisfy positive or negative correlations. A closed-form solution is obtained. Some numerical calculation examples are given, for which we get more direct view of the relationship among the parameters. The graph of the model shows that the inflection impacts the LCDS spread more than the interest does.

\section{References}

Ahn, D. H. \& Gao, B. (1999). A parametric nonlinear model of term structure dynamics, Review of Financial Studies, 12, 721-762. http://dx.doi.org/ 10.1093/rfs/12.4.721

Dobranszky P. \& Schoutens W., (2008). Generic Levy One-Factor Models for the Joint Modeling of Prepayment and Default: Modeling LCDX, Technical Report 08-03, Section of Statistics, K.U. Leuven. http://dx.doi.org/ 10.1.1.139.5613

Dobranszky, P. (2008). Joint Modeling of CDS and LCDS Spreads with Correlated Default and Prepayment Intensities and with Stochastic Recovery Rate, Technical Report 08-04, Section of Statistics, K.U. Leuven. http://dx.doi.org/ 10.1.1.139.6181

Duffie, D. (2005). Credit risk modeling with affine processes. Journal of Banking and Fi-nance. http://dx.doi.org /10.1016_j.jbankfin.2005.02.006

Hurd, T. R. \& Kuzneton, A. (2008). Explicit formulas for Laplace transforms of stochastic integrals, Markov Process. Related Fields, 14, 277-290.

Liang, J. \& Wang, T. (2012). Valuation of a loan-only credit default swap with negatively correlated default and prepayment intensities, to appear in International Journal of Computer Mathematics.

Liang, J. \& Zhou, Y. (2011). Valuation of a tranched Loan Credit Default Swap Index, Technology and Investment, 
PP.240-246. http://dx.doi.org/ 10.4236/ti.2011.24025

Qian, X., Jiang, L. \& Xu, C. (2008). Explicit formulas for pricing of Mortgage-Backed Securities in a case of prepayment rate negatively correlated to interest, Mathematic Finance.

Shek, H., Uematsu, S. \& Wei, Z. (2007). Valuation of Loan CDS and CDX, working paper, Stanford University

Wei, Z. (2007). Valuation of Loan CDS under Intensity Based Model, working paper, Stanford University. http://dx.doi.org/ 10.1.1.139.4910

Wu, S., Jiang, L. \& Liang, J. Pricing of Mortgage-Backed Securities with Repayment Risk, to appear in International Journal of Theoretical and Applied Finance

Zhou, Y. \& Liang, J. (2010). Valuation of a Basket Loan Credit Default Swap, International Journal of Financial Research.21-29. http://dx.doi.org/ 10.5430/ijfr.v1n1p21
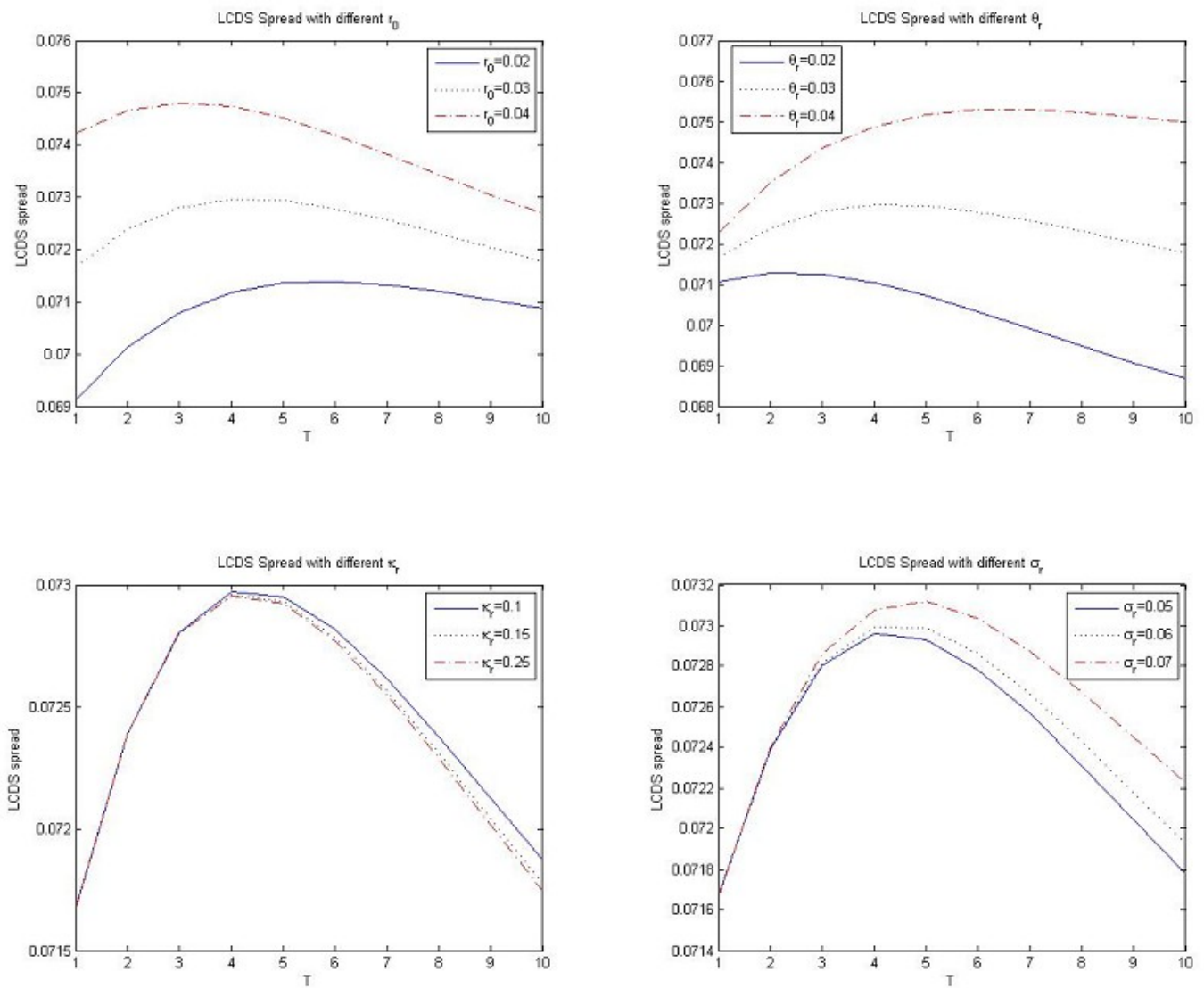

Figure 1. Term structure of the LCDS spread for different $r$. 

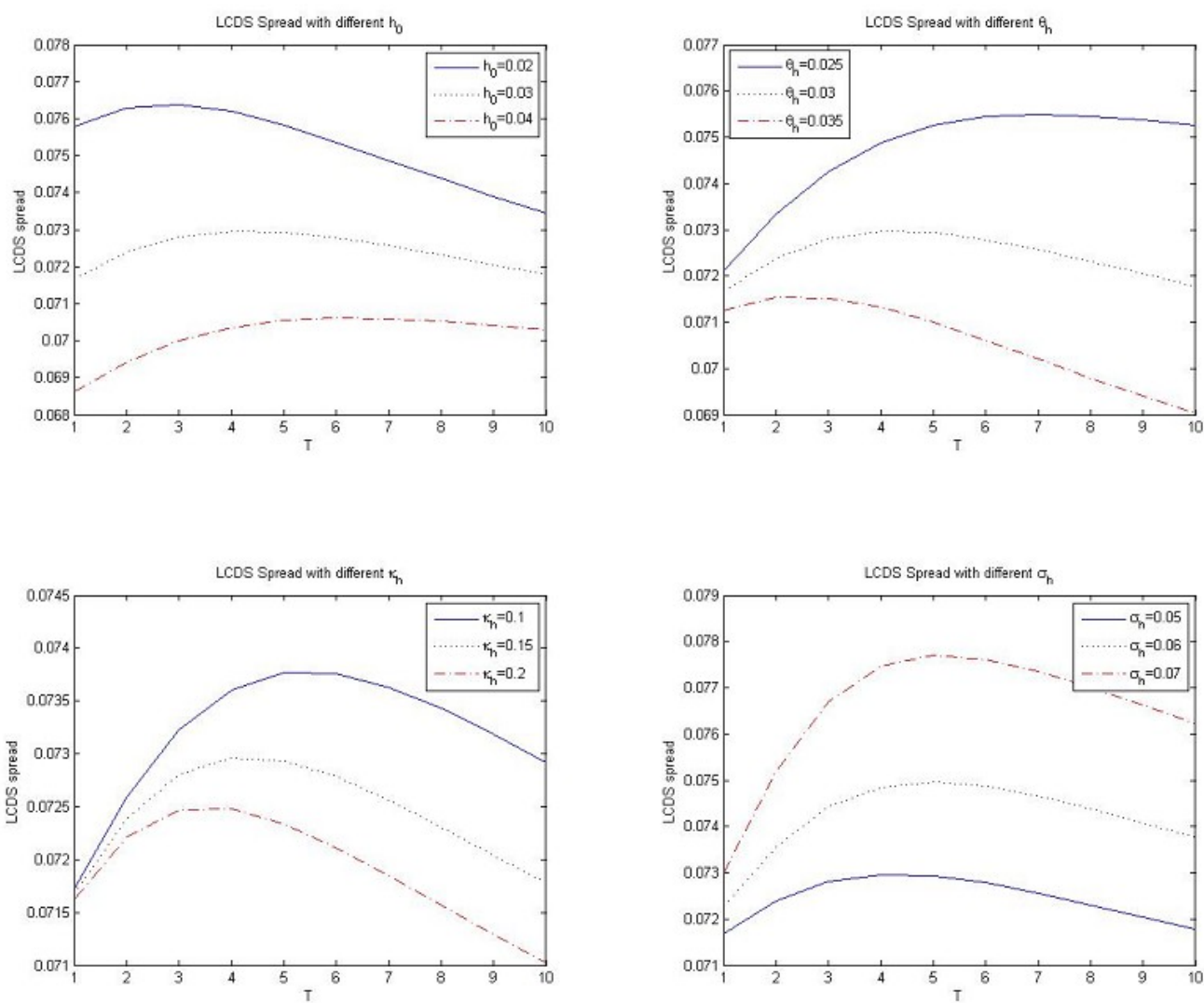

Figure 2. Term structure of the LCDS spread for different $h$

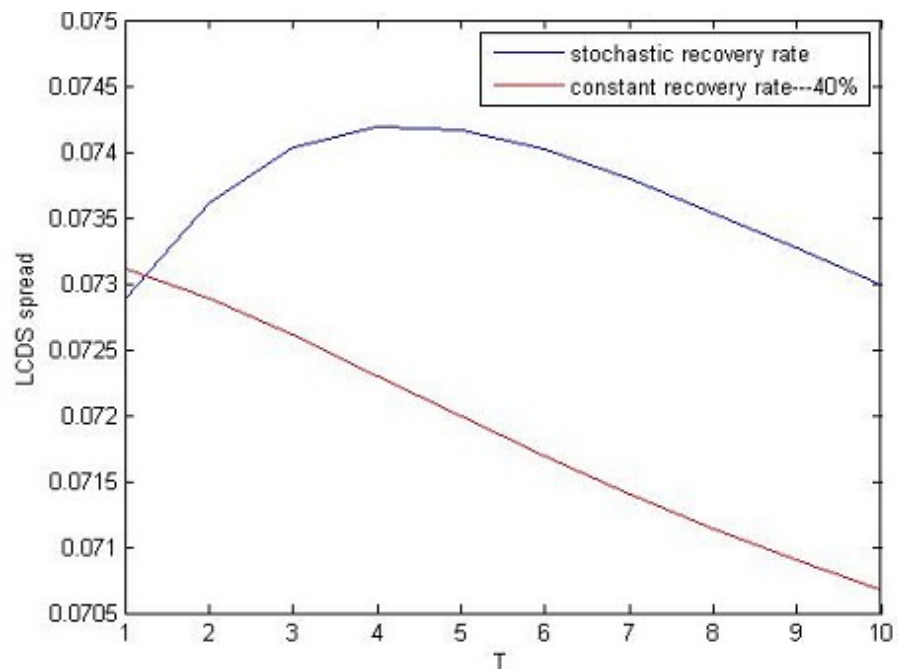

Figure 3. LCDS spread with different recovery rate. 